\title{
Las tumbas de los reyes visigodos: un problema histórico
}

\section{The tombs of the Visigothic kings: a historical problem}

\author{
JAVIER ARCE \\ Université de Lille III-Charles de Gaulle. HALMA-UMR 8164 \\ Pont de Bois, BP 60149, F-59653 Villeneuve d'Ascq Cedex \\ j.arce@wanadoo.fr
}

Resulta un fenómeno curioso el hecho de que no conozcamos nada de las tumbas de los reyes visigodos en Hispania durante su estancia de más de dos siglos en la Península. Ningún cronista ni historiador contemporáneo hace mención de ello y no hay testimonios arqueológicos de ningún panteón, tumba o sarcófago de ningún rey. La explicación podría estar en el peculiar carácter de la monarquía visigoda, que no es hereditaria y que, en tanto en cuanto sometida férreamente al dictado de la Iglesia, debe practicar y expresar públicamente la humilitas también en sus formas de enterramiento. No existe panteón real porque no es una monarquía aglutinante de un pueblo.

PALABRAS CLAVE

MONARQUÍA VISIGODA, TUMBAS REALES VISIGODAS, TOLETUM

It is a strange fact that we know nothing about the tombs of the Visigothic kings in Hispania during their presence in the Peninsula for more than two centuries. No contemporary chronicler or historian mentions them and there is no archaeological evidence for any pantheon, royal tomb or sarcophagus. An explanation for this could lie in the peculiar nature of the Visigothic monarchy which was nonhereditary and, because of its submission to the iron rule of the Church, was also obliged to practice and publically express its humilitas in its forms of burial. There was no royal pantheon because it was not a monarchy that was tied to the people.

\section{KEYWORDS}

VISIGOTHIC MONARCHY, VISIGOTHIC ROYAL TOMBS, TOLETUM 
El rey godo Atanarico fue a la capital Constantinopla a negociar con el emperador Teodosio en el año 381 y le sorprendió la muerte en la ciudad inopinadamente. El historiador Jordanes (Getica, 28 y 144) cuenta que el emperador romano le dio una sepultura digna de su rango (rex) y marchó él mismo a la cabeza del cortejo fúnebre en señal de homenaje. Tanto Zósimo (IV, 34,4-5) como Amiano Marcelino (27, 5,10) confirman la noticia. Pero este último añade un detalle que me parece significativo: el historiador señala que Atanarico fue sepultado con magnifico boato "según el ritual romano», ritu nostro. Amiano está enfatizando aquí la diferencia entre los funerales dados por los godos a sus reyes o líderes y los ofrecidos por los romanos a sus gobernantes/emperadores. Este boato, esta parafernalia, que consistía en la ceremonia del funus publicum o funus imperatorum, estaba integrado por la procesión por las calles de la ciudad hasta la tumba o mausoleo, por la presencia del ejército y representantes de todos los órdenes sociales, la laudatio, o en época cristiana la consolatio, algo que era por completo ajeno al pueblo godo y, por ello, tanto Zósimo como Amiano y Jordanes, destacan la impresión estupefacta de los seguidores de Atanarico ante un tal homenaje público por parte de Teodosio, porque para ellos era un espectáculo inusual, diferente y novedoso (Arce, 1988). Los funerales de los reyes godos y los funerales de los emperadores romanos eran completamente diferentes.

Después de este funeral espectacular «a la romana», entra en escena otro bien conocido, el de Alarico. Pero no es fácil afirmar que este fuera un funeral típicamente "godo».

El historiador Jordanes, también, es el único que nos informa con detalle del enterramiento de Alarico, fallecido en Cosenza hacia el otoño del año 410. La descripción es bien conocida (Arce, 2018: 141-155). Muerto el rex se ordena a una serie de esclavos desviar el curso del río Busento, que pasa por Cosenza, se entierra a Alarico y luego se vuelve a hacer pasar el rio por su curso normal. Los encargados de la operación fueron inmediatamente asesinados a fin de que nadie pudiera desvelar el lugar donde había sido enterrado. Jordanes añade que Alarico fue enterrado cum multis opibus, resultado de sus razias por toda Italia y el saqueo de Roma en el verano de ese mismo año.

Muchas preguntas surgen ante esta descripción, hecha por un historiador que escribe casi ciento cincuenta años después del hecho, en época de Justiniano. ¿Hasta qué punto esta descripción no es una invención de Jordanes? ¿De dónde sacó esta información? ¿Por qué no trasladar el cadáver a su tierra natal? ¿Qué paralelos existen con este tipo de enterramiento? ¿Es propio de los godos ser enterrados en el lecho de los ríos? ¿Por qué enterrarlo con sus tesoros? ¿Cuáles eran estos? El modo de enterramiento de Alarico ¿marcó una tradición en el mundo godo e inició una costumbre seguida posteriormente por sus sucesores en la dirección del pueblo godo?

De ser cierta la descripción de Jordanes sobre el enterramiento de Alarico, el hecho fue excepcional y no tuvo continuidad. Enterramientos semejantes se encuentran en la descripción de Dion Casio de la muerte del rey de los dacios, Decébalo, en el siglo II $(78,14$, 4 y 5). Algunos autores, como Herwig Wolfram, piensan que el de Alarico es un tópico (Wolfram, 1985: 248). Muerto Alarico las circunstancias no permitían que su cadáver fuera 
trasladado con seguridad a su lugar de origen, una isla del Danubio, y además no existía una capital, una sede o residencia del rey godo. Con Alarico, sus seguidores son un grupo en movimiento que busca instalarse y establecerse definitivamente. No existe para ellos un "centro de poder".

El de Atanarico fue un funeral romano. El de Alarico, un funeral excepcional o una invención de Jordanes que escribe —no lo olvidemos- en época de Justiniano. Por tanto, nos quedamos sin saber nada de los funerales de los reyes godos en este primer período. El momento en que ambos tienen lugar corresponde al período arriano de los godos.

De cuando los godos o, mejor, el conglomerado de gentes que seguían a Alarico y luego a Ataúlfo, llegan a Aquitania y se establecen en Tolosa según el foedus de 418, tampoco sabemos nada sobre sus costumbres de funerales regios ni de sus tumbas. Solo hay un episodio que hay que recordar, pero que, por su carácter especial, es poco significativo: el hijo de Ataúlfo y de Gala Placidia — una cristiana-muerto prematuramente, fue enterrado, nos dice Olympiodoro (Frag. 26), en un pequeño sarcófago en un oratorio de Barcino. La religión de la madre, ferviente católica, se muestra en este caso decisiva.

A partir de este momento hay un vacío en los textos - literarios, cronísticos, conciliares- que no dicen nada nunca ni se ocupan de los enterramientos de los reyes que se suceden en el Reino Visigodo. Este es un problema fundamental para el propósito de lo que voy plantear aquí.

Ninguno de los reinos de la Península dispuso de un panteón dinástico estable hasta la organización por la casa de Austria de uno en el monasterio de El Escorial, subraya Raquel Alonso (2013: 136).

No pretendo aquí revisar todas las hipótesis y teorías que se han formulado sobre los posibles enterramientos de los reyes visigodos. Raquel Alonso Álvarez ha escrito varios artículos muy completos y documentados sobre el tema (Alonso, 2005 y 2008). Sus conclusiones son tajantes: no hay ninguna evidencia segura de dónde estuvieron enterrados los reyes godos, excepto en tres casos, que tampoco están completa y definitivamente confirmados por las fuentes, tanto arqueológicas como literarias. Son los casos de Chindasvinto, Recesvinto y Wamba. Del resto no podemos decir nada preciso o que no se remonte, en todo caso, a fuentes medievales no anteriores al siglo x, en plena Edad Media. Y ese es el problema intrigante: ¿por qué un cronista/historiador como Isidoro no expresa donde fueron enterrados? Pero no es solamente Isidoro, ninguna fuente ni cronista de los siglos VI y vII precisa el hecho. Esto contrasta con los historiadores o cronistas romanos que no dejan de señalar que un emperador murió en tal sitio y fue enterrado o trasladado después a su mausoleo en Roma o en Constantinopla. Como dice Raquel Alonso (2013: 137), «los escritores hispanovisigodos obviaron la muerte de los reyes». Yo diría, mejor, que se olvidaron del lugar de enterramiento de los reyes, porque historiadores o cronistas como Isidoro no dejan de señalar dónde murieron y las circunstancias de su muerte, pero no dicen nada a propósito del lugar de enterramiento.

Si analizamos con algún detalle las informaciones que poseemos, se pueden observar algunos hechos significativos. 


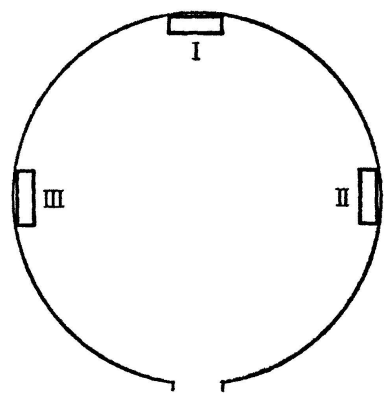

Stage $A$

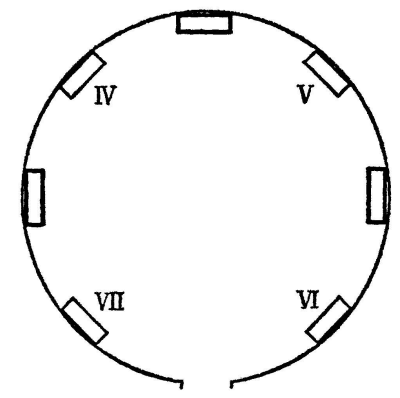

Stage $B$

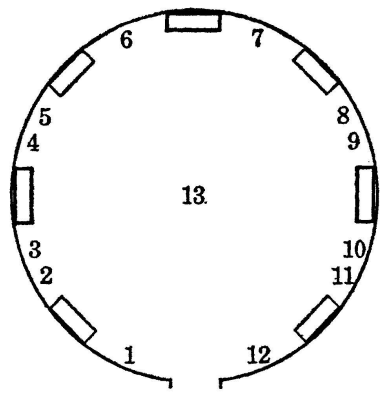

Stage $C$

Figura 1. Plano esquemático del mausoleo de Constantino (según P. Grierson, 1962: 23).

Hasta Atanagildo (568) los reyes que están en la Península no tienen una sede fija y les sorprende la muerte, violenta o natural, en distintos escenarios. Teudis murió en palacio, in palatio vulneratus, pero Isidoro no precisa más. Teudiselo murió durante un banquete en Hispalis, inter epulas iugulatur Hispali. Agila fue asesinado en Emerita. Atanagildo falleció en Toledo y, a partir de ese momento, a muchos reyes les sorprendió la muerte en la urbs regia o sedes regia: Leovigildo, de muerte natural, Recaredo, morte propia; Gundemaro, también de morte propia en Toledo, así como Sisebuto, Chintila, Tulga, Ervigio, Egica y, probablemente, Witiza. De aquellos que murieron de muerte violenta o como resultado de un complot, no podemos esperar que fueran enterrados ni en una tumba digna ni con el ceremonial acostumbrado. Este fue seguramente el caso de Witerico, que murió en un banquete, nos dice Isidoro, y corpus eius viliter est exportatum atque sepultum. De otros, simplemente no tenemos ninguna noticia. De tres reyes sabemos que no murieron en Toledo: Chindasvinto, Recesvinto y Wamba. Y fueron enterrados lejos de la ciudad (Alonso, 2008 y 2013). Y a ellos me referiré brevemente más adelante. El caso de Rodrigo, que murió o desapareció en la batalla de Guadalete en 711, es muy dudoso, aunque recientemente se ha mantenido que fue enterrado en Viseu, en el actual Portugal.

Por todo ello cabría esperar que en Toledo hubiese un mausoleo, un panteón para enterrar allí a los reyes. Un mausoleo que fuese aglutinante de una memoria histórica de los distintos gobernantes que se sucedieron.

Se ha dicho siempre que una de las características de los visigodos fue la imitatio imperii. Ya Sánchez Albornoz (1971: 191) lo había observado: "Conocemos el espíritu simiesco y el gusto por el remedo de los modelos romanos de la corte visigoda». La imitación alcanza, efectivamente, aspectos de la organización administrativa, legislativa y, eventualmente, el ceremonial. Hay quienes incluso han sugerido que Toledo tiene como modelo el urbanismo y las iglesias de Constantinopla (Martin, 2003: 117), una idea que no resiste un análisis comparativo entre ambas ciudades.

Sin embargo, desde Constantino (fig. 1), los emperadores se entierran en el mausoleo de Constantinopla, que será la capital oriental. No todos, es cierto. Hay excepciones: 
Figura 2. Sarcófago de pórfido atribuido a Constantino, jardines del Istanbul Archaeological Museums (foto: pomona.edu).

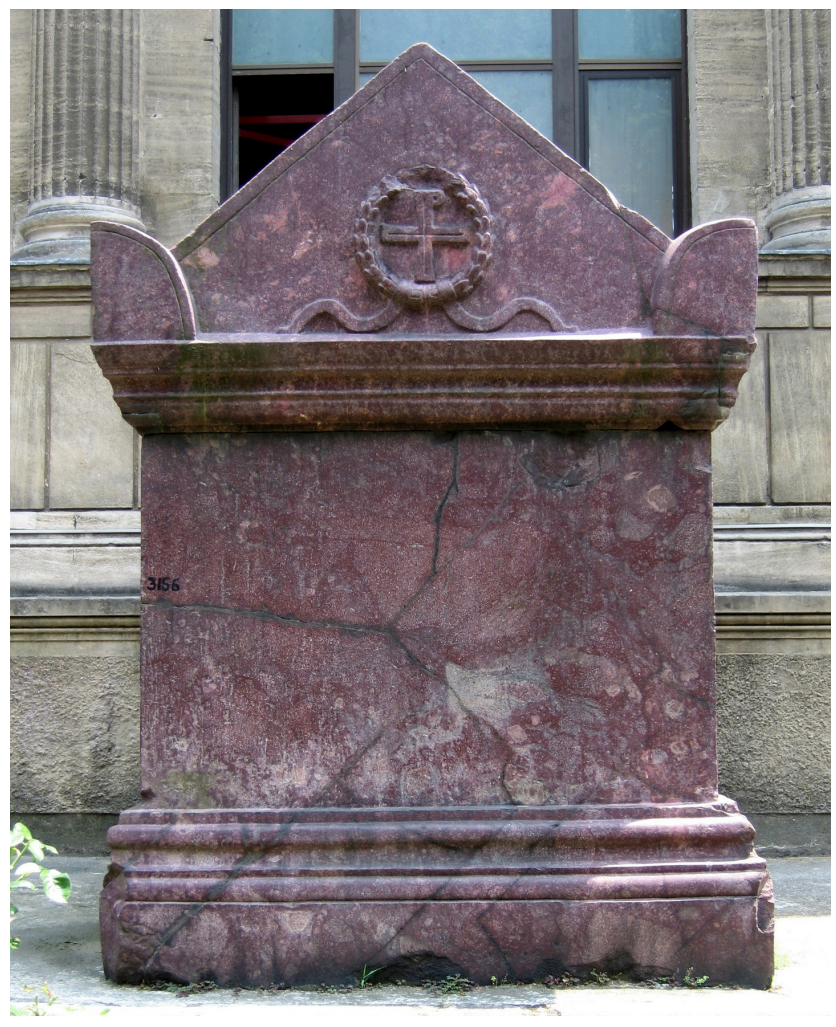

Honorio, por ejemplo, que fue enterrado en Roma, cerca de la tumba de Pedro; otros, cuyo cuerpo no se encontró debido a una muerte violenta o por haberse perdido en un rio, no encuentran sepultura en el mausoleo.

El enterramiento de los emperadores después de Constantino se hace en el mausoleo circular comenzado por el primer emperador cristiano y terminado por su hijo y sucesor, Constancio II (Mango, 1991: 24-25) (fig. 2). Allí fue sepultado incluso el pagano Juliano, que fue trasladado desde Tarso, donde fue primeramente enterrado al regreso de las tropas después de la expedición fracasada contra los sasánidas, por su sucesor Joviano. Luego fue desplazado a la ciudad de Constantinopla (Arce, 1984).

Numerosas fuentes registran el señalamiento del lugar del enterramiento en el mausoleo, tanto contemporáneas como más tardías (Arce, 1994: 148-149). En la literatura antigua no faltaron intentos de hacer un Catalogus sepulchrorum o, mejor, un Necrologium imperatorum. De hecho, el emperador bizantino Constantino Porphyrogeneta (siglo x) mandó compilar un catálogo de esta naturaleza que fue copiado después de su muerte y que constituyó una especie de apéndice de su libro De Cerimoniis. La lista obedece a la intención de registrar las tumbas de los emperadores enterrados en el mausoleo y en 
la iglesia adyacente. Este catálogo no es el único, y de semejante valor es el Necrologium imperatorum o la lista que ofrece el historiador bizantino Nicolas Mesarites. Este tipo de recopilación tiene sus antecedentes en la lista ofrecida por Eusebio de Cesarea y Jerónimo en su Chronica, conservada solo en su versión armenia (Downey, 1959; Grierson, 1962; Arce 1988: 116 y ss.).

Pero, como he dicho, no encontramos en las fuentes de época visigoda ninguna referencia ni interés alguno por mencionar dónde estaban enterrados los reyes. En el tema del mausoleo común no hay imitatio imperii y parece que Constantinopla no sirve de modelo para los obispos o reyes. Pensemos especialmente en Leovigildo, que según Isidoro fue quien primero introdujo el ceremonial bizantino y la imitación de los modelos de la corte de Constantinopla. Varios autores lo afirman sin reservas: «al menos desde la época de Leovigildo el ceremonial real siguió estrechamente el mismo contemporáneo ejemplo del Oriente romano" (Heather, 1996: 293). Resulta sorprendente que, si la corte visigoda sigue el ceremonial bizantino, no incluya también el ceremonial funerario y el mausoleo.

Y el ceremonial bizantino para los funerales del emperador era un ritual que todavía tenía mucho de la tradición romana, como se demuestra en las exequias de Justiniano, según relata el poeta Flavius Cresconius Coripus (III, I-61) en su alabanza de Justino. ${ }^{1}$ El cadáver de Justiniano yacía en el palacio en un féretro de oro elevado sobre un pedestal, coronado con la diadema y vestido de púrpura - como el de Constantino- según la descripción de Eusebio, diademate comptus purpureaque in veste iacens. Justino, el sucesor en el Imperio, llega ante el féretro y habla al cadáver de Justiniano, tal y como ocurrió en el funeral de Pertinax. Los senadores esperaban bajo los pórticos. Se quemó el incienso — se echa miel olorosa y ungüentos exóticos a fin de preservar el sagrado cuerpo para siemprecomo se hizo con el cadáver de Poppea o de Herodes, conservados en miel «a la manera oriental». Pero en la translatio de Justiniano no hay effigies cerea, ni está ya el ejército, sino una venerable fila de diáconos cantores y un coro de vírgenes. El pueblo acompaña con antorchas encendidas. Si bien estas no faltaban en la pira funeraria romana pagana o en la procesión nocturna, aquí y ahora, con Justiniano, su sentido simbólico ha cambiado: representan la luz eterna y perpetua de la resurrección.

Pero volvamos a los visigodos en Hispania. Durante bastante tiempo se ha pensado que el panteón real, el mausoleo real visigodo, era la iglesia de Santa Leocadia, ${ }^{2}$ que como panteón de los reyes presenta cierta problemática. En primer lugar, no sabemos si hubo en Toledo una, dos o tres iglesias dedicadas a la mártir patrona de la ciudad. En segundo lugar, en el sitio donde se dice que estaba situada la iglesia, in suburbio, que muchos identifican como la zona de la llamada Vega Baja, fuera de las murallas, no se han encontrado restos claros de una iglesia ni sarcófagos ni nada que permita identifi-

1. Flavius Cresconius Corippus, In laudem lustini Augusti minoris, Libri VI, ed. A. Cameron, Londres, 1976.

2. Véanse los argumentos utilizados por la historiografía que desautorizan tal hipótesis en Velázquez y Ripoll, 2000 : 557, y Alonso, 2013: 139. 
car el monumento; en cualquier caso, las excavaciones allí realizadas son incompletas. Algunas fuentes literarias nos dicen que en Santa Leocadia estaba enterrado el obispo Ildefonso de Toledo y, según el falso Chronicon atribuido a Liutprando de Cremona, que pasó algún tiempo en Toledo antes de desplazarse a la corte de los otones y de hacer su famoso viaje diplomático a la corte de Bizancio, y que escribe, hacia 960, que dos reyes estaban enterrados en Santa Leocadia: Sisenando y Witiza, una noticia que podría ser verídica dada la presencia transitoria de Liutprando en Toledo, aunque el Cronicon Liutprandi puede ser una falsificación (referencias en Alonso, 2013: 141). Pero dos reyes no hacen un panteón o mausoleo real. También la Crónica de Rasis o «Crónica del moro Rasis» (889-955), dice que Recaredo fue enterrado en Santa Leocadia, pero sabemos que esto es imposible, ya que la iglesia fue fundada por el rey Sisebuto en 618 y que Recaredo murió en 601. Por ello conviene recordar aquí lo que dice Raquel Alonso (2003: 363): «esta idea (la de la existencia de un panteón o mausoleo de los reyes visigodos en Santa Leocadia) ha sido capaz de imponerse incluso a la absoluta carencia de testimonios que prueben su existencia».

Como he dicho antes, tres casos atestiguan el enterramiento de tres reyes en otros lugares distintos de Toledo.

Ildefonso de Toledo nos dice que Recesvinto murió en su villa de Gérticos, in villa propia; Chindasvinto, en San Roman de Hornija (Valladolid), y Wamba, en Pampliega (Valladolid) (¿en un monasterio?) (Alonso, 2008). A pesar de que las fuentes sobre estos enterramientos son todas muy tardías, excepto Ildefonso, las tres noticias hablan en favor de que los reyes pudieran enterrarse en sus villae, de la misma manera que en época romana los propietarios se enterraban en el territorio de sus propias villae o, en época cristiana, en un monasterio (Bodel, 1997).

Al fin y al cabo, el hecho de saber con certeza si están enterrados aquí o allá no es tan importante como el hecho de que no haya ninguna mención a sus lugares de enterramiento y a que no exista noticia sobre ello. Este es —en mi opinión- el problema histórico.

¿Por qué este silencio? ¿Por qué este olvido o este desinterés? ¿O es que los funerales de los reyes visigodos no tenían nada de excepcional y eran como los de personas aristocráticas y no tenían un mausoleo específico? ¿Podemos encontrar alguna respuesta a estas preguntas? Probablemente no de manera definitiva, pero sí, al menos, y esta es la obligación del historiador, de manera tentativa, quizás hipotética, a modo de propuesta.

Pienso que el problema puede que resida precisamente en el carácter mismo de la realeza visigoda en Hispania.

Janet Nelson ha escrito acertadamente que «las tumbas de los gobernantes permanecen como un potente símbolo de la identidad nacional justo como en la Edad Media las tumbas de los reyes se convierten frecuentemente en lugares focales de culto dinástico y monárquico» (Nelson, 2000: 132). Ciertamente no podemos aplicar estas ideas a los reyes visigodos de Hispania. No son representativos de la identidad nacional ni poseen lugares de culto dinástico. 
Solamente cuando se introduce en el Reino Visigodo la ceremonia de la santa unción, fecha que se discute y se sitúa entre Sisenando (631-639) y un poco antes de Wamba (672-680), pudiéramos pensar que la figura del rey se convierte en sacra, ungida por Dios. A una tal realeza correspondería un funeral exaltador y una tumba respetuosa y memorable digna de ser visitada y de culto. Pero ni siquiera después de Wamba tenemos evidencia de este hecho en el mundo visigodo en Hispania, lo que quiere decir que el rey es ungido y sagrado, por tanto, solo mientras está vivo.

La exaltación en el funeral, sus preparativos, la representación que implica, responden a la legitimación del heredero en presencia de la aristocracia y del pueblo. Pero el rey visigodo ni necesita la legitimación del heredero, ni es un santo, ni un isoapostolos, como Constantino, ni un dios; es un elegido en vida, sometido a la Iglesia, pero tras la muerte - $\mathrm{O}$, mejor, a su muerte - es un simple mortal. Y, como tal, debe ser enterrado con simplicidad, con humildad. Esta idea de la humildad, que constituye una de las características de los reyes, está señalada como una virtud de los mismos, tal y como determina el IV Concilio de Toledo: entre sus virtudes debe destacar, en primer lugar, la humilitas, y luego la moderatio, la iustitia, la pietas erga subditos y la indulgentia (Vives, 1963: tol. IV, 75, II). A esta idea corresponden los enterramientos in porticum ecclesiae sepultus, que es el caso de Wamba y del rey Pipino en Saint Denis.

El problema de la sucesión al trono entre los visigodos es que este no es hereditario, excepto en algunos casos puntuales, y por lo tanto no se necesita legitimar el poder de la familia delante de la aristocracia que ha dado su consenso.

La costumbre - mos - de los reyes visigodos en los funerales tenía una tradición de la que, como hemos dicho, no sabemos nada. Probablemente era sencilla y sin ceremonias especiales o tumbas asociadas espectaculares. A esta conclusión llega también Alain Dierkens en su análisis del funeral de Carlomagno (Dierkens, 1991: 145-180; cf. también Nelson, 2000: 145 y ss.). Dierkens señala, como diferencias entre los funerales de Bizancio y los de Occidente, hablando de la tumba de Carlomagno en Aquisgrán, que los de Occidente no tienen un ritual desarrollado, no existe una iglesia panteón dinástica, ni una tumba espectacular que sea de veneración especial; no hay preparación del cuerpo. El Concilio de Valencia de 549 indica que los obispos se deben enterrar el mismo día de su muerte, mox sepulturae tradat (Vives, 1963: val., c. IV) y Carlomagno fue también enterrado el mismo día de su fallecimiento, y no son sepultados con insignia. Sin embargo, el enterramiento en un sarcófago traído de Italia para Carlomagno — con la representación de Proserpina y el dios de los infiernos- (fig. 3) es una prueba de que, en esto, si que se siguió el modelo imperial, la imitatio imperii.

No hay imitatio imperii en este caso entre los visigodos, a pesar de que hablamos tanto de su imitación del emperador y el ceremonial bizantino. No hay una conciencia de la realeza unificadora. Toledo es la urbs regia por que el rey habita allí y allí tiene - a veces- su residencia. Pero su tumba no es un lugar de culto a la realeza o de veneración unificado. Esto explica que los historiadores y cronistas eclesiásticos no hagan ninguna alusión a ello. 


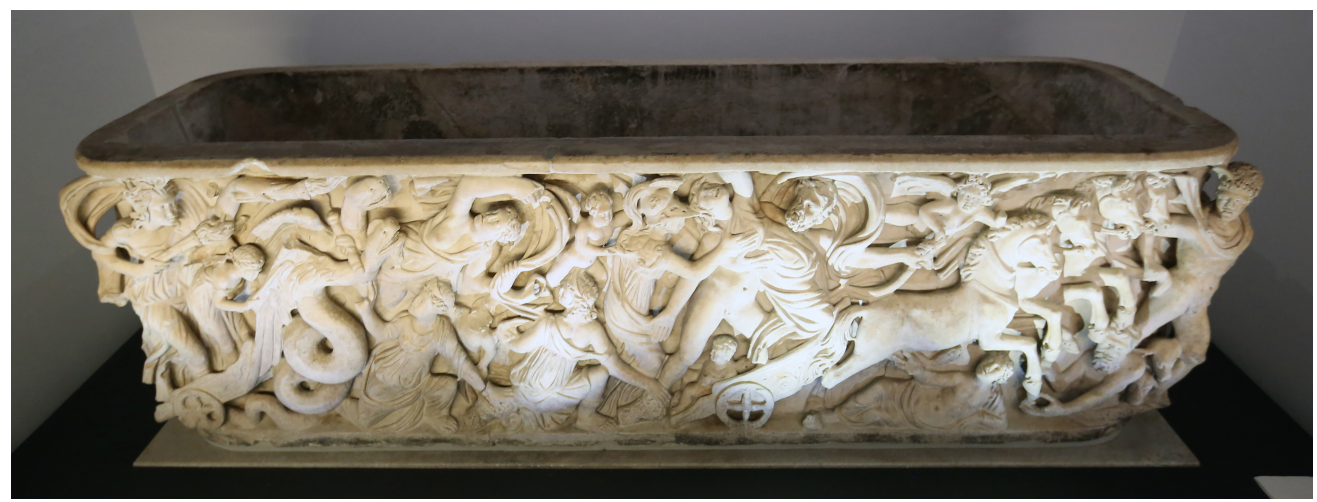

Figura 3. Sarcófago de Carlomagno en la catedral de Aquisgrán (foto: Domschatzkammer Aachen).

La imitatio imperii es patente en la administración, en las leyes, en algunas celebraciones triunfales, pero no sé hasta qué punto en lo que se refiere al ceremonial; no es una creación de Isidoro que trata de identificar a Leovigildo con emperador romano.

Precisamente por todo lo dicho es por lo que las tumbas de los reyes visigodos $-\mathrm{y}$ su eventual panteón o mausoleo, que parece que no existió-, y que han sido silenciadas por las fuentes contemporáneas, son un problema histórico. Y quizá seguirá siéndolo durante mucho tiempo.

\section{Bibliografía}

ALONSO, R., 2005, Los enterramientos de los reyes visigodos, en Fundamentos medievales de los particularismos hispanos, IX Congreso de Estudios Medievales, Fundación Sánchez Albornoz, León, 363-375.

ALONSO, R., 2008, Hornija, Bamba, Pampliega: las elecciones funerarias de los reyes hispanovisigodos, Territorio, Sociedad y Poder 3 , 13-27.

ALONSO, R., 2013, Las sepulturas de los reyes godos en Hispania: Chindasvinto, Recesvinto y Wamba, Pyrenae 44.1, 135-155.

ARCE, J., 1984, La tumba del emperador Juliano, Lucentum 3, 181-191.
ARCE, J., 1988, Funus imperatorum. Los funerales de los emperadores romanos, Madrid, Alianza.

ARCE, J., 1994, Constantinopla, Tarraco y Centcelles, Butlletí Arqueològic 16, 147-165.

ARCE, J., 2018, Alarico. La integración frustrada, Marcial Pons Historia, Madrid.

BODEL, J., 1997, Monumental villas and villa Monuments, Journal of Roman Archaeology 10, 5-35.

DIERKENS, A., 1991, Autour de la tombe de Charlemagne : considérations sur les sépultures et les funérailles des souverains carolingiens et des membres de leur famille, Byzantion 61.1, 156-180. 
DOWNEY, G., 1959, The Tombs of the Byzantine Emperors at the Church of Holy Apostles in Constantinople, Journal of Hellenic Studies 79, 27-51.

GRIERSON, P., 1962, The Tombs and Obits of the Byzantine Emperors (337-1042), with an additional note by C. Mango and I. Ševčenko, Dumbarton Oaks Papers 16, 3-63.

HEATHER, P., 1996, The Goths, Oxford.

MANGO, C., 1991, Le développement urbain de Constantinople (IV ${ }^{e}$-VII ${ }^{e}$ siècles), París.

MARTIN, C., 2003, La géographie du pouvoir dans l'Espagne wisigothique, Presses Universitaires du Septentrion, Lille.

NELSON, J. L., 2000, Carolingian royal funerals, en F. THEUWS y J. L. NELSON (eds.), Rituals of
Power. From Late Antiquity to the Early Middle Ages, Transformation of the Roman World 8, Brill, Leiden-Boston-Colonia, 131-184.

SÁNCHEZ ALBORNOZ, C., 1971, El aula regia y las asambleas políticas de los godos, en Estudios visigodos, Istituto Storico Italiano per il Medio Evo, Roma, 150-252.

VELÁZQUEZ, I. y RIPOLL, G., 2000, Toletum, la construcción de una urbs regia, en G. RIPOLL y J.M. GURT (eds.), Sedes regiae (ann. 400-800), Reial Acadèmia de Bones Lletres, Barcelona, 521-578.

VIVES, J., 1963, Concilios visigóticos e hispanorromanos, con la colaboración de T. Marín y G. Martínez, Barcelona-Madrid.

WOLFRAM, H., 1985, Storia dei goti, Roma, 1985 (trad. italiana de M. Cesa). 